\title{
Temporal characterization of a plasma-based seeded XUV laser using a laser-dressed photoionization technique
}

L. Dakroub, T. Sinyakova, D. Cubaynes, A. K. Pandey, M. Pittman, et al.

L. Dakroub, T. Sinyakova, D. Cubaynes, A. K. Pandey, M. Pittman, E. Baynard, C. Bourassin-Bouchet, O. Guilbaud, A. Klisnick, "Temporal characterization of a plasma-based seeded XUV laser using a laser-dressed photoionization technique," Proc. SPIE 11886, International Conference on XRay Lasers 2020, 118860N (8 July 2021); doi: 10.1117/12.2594657 


\title{
Temporal characterization of a plasma-based seeded XUV laser using a laser-dressed photoionization technique
}

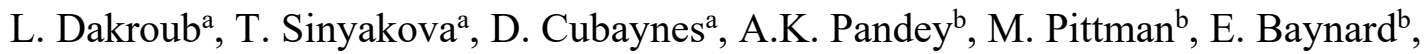 \\ C. Bourassin-Bouchet ${ }^{\mathrm{c}}$, O. Guilbaud ${ }^{\mathrm{b}}$, and A. Klisnick ${ }^{\mathrm{a}}$ \\ a ISMO, Université Paris-Saclay, CNRS, 91405 Orsay, France; ${ }^{\mathrm{b}}$ IJC-Lab, Université Paris-Saclay, \\ CNRS, 91405 Orsay, France ; ' LCF, Institut d'Optique Graduate School, CNRS, Université Paris- \\ Saclay, 91127 Palaiseau, France
}

\begin{abstract}
We report on the development and implementation of a diagnostic for the temporal characterization of seeded XUV laser pulses, based on laser-dressed photoionization in the sideband regime, using a home-made velocity map-imaging spectrometer as the central element. The diagnostic was recently tested at the LASERIX facility with the seeded Ne-like titanium laser at $38 \mathrm{eV}$ as the XUV source, overlapped with an infrared pulse of variable duration and intensity.
\end{abstract}

Keywords: seeded XUV-laser, photoelectron spectroscopy, temporal characterization, laser-dressed photoionization, sidebands.

\section{INTRODUCTION}

Plasma-based XUV lasers operated in the seeded $\operatorname{mode}^{1}$ have led to significant improvements of their beam properties, in particular in terms of coherence or wavefront. On the other hand numerical simulations suggest that a better control of the seeding conditions is a promising way to achieve ultra-short, ultra-intense amplified pulses, below the picosecond timescale limit imposed by the amplifier spectral width ${ }^{2}$. Progress towards this goal requires that reliable temporal diagnostics, able to characterize the XUV pulse temporal profile with femtosecond resolution, possibly in a single-shot, become available. The development of such temporal diagnostics is also a current challenge for other high-brightness, ultrashort XUV sources, namely free-electron lasers ${ }^{3}$ and high-order harmonics generated from gas or from plasma mirrors ${ }^{4}$.

Among the different methods which have been proposed or tested, those based on the photoionization of atoms in a gas by the XUV pulse in the presence of a laser-dressing field, have become the most extensively explored. In the presence of the dressing field the distribution of kinetic energy of the released photoelectrons is modulated in a way that is controlled, in particular, by the relative magnitude of the dressing field period $T_{d}$ with respect to the duration $\tau_{X U V}$ of the XUV pulse ${ }^{5}$. If $\tau$ XUV $<<T_{d}$ the photoline induced by the XUV photons is either shifted or broadened (streaked), depending on the relative timing between the XUV pulse and the temporal phase of the dressing field. Temporal information about the XUV pulse can be retrieved in a single-shot from the streaked spectrum. The streaking technique has been successfully demonstrated with XUV free-electron lasers, using a THz dressing pulse ${ }^{6}$. If $\tau_{\mathrm{XUV}}>>\mathrm{T}_{\mathrm{d}}$ satellite peaks called sidebands appear on each side of the main photoline, when the XUV pulse and the dressing pulse are overlapped in time. Each sideband corresponds to the absorption or stimulated emission of one or more dressing photons and is separated from the next one by exactly the dressing photon energy. By measuring the variation of the sidebands intensity while varying the delay between the XUV pulse and the dressing pulse, a cross-correlation signal is obtained from which the temporal profile of the XUV pulse can be retrieved, if the profile of the dressing pulse is known. The sideband technique has been used to measure the duration of $\mathrm{XUV}^{7}$ and $\mathrm{X}-\mathrm{ray}^{8}$ free-electron laser (XFEL) pulses, as well as of high-order harmonic $(\mathrm{HH})$ radiation generated from gas ${ }^{9}$, using an infrared (IR) dressing field in the femtosecond range. The generation of sidebands is also at the heart of more elaborate techniques such as RABBIT ${ }^{10}$, or FROG$\mathrm{CRAB}^{11}$, with which temporal characterization in the attosecond range has been demonstrated.

The main goal of our work is to implement the technique of laser-dressed photoionization in both the sideband (IR dressing) and the streaking ( $\mathrm{THz}$ dressing) regimes, for the temporal characterization of seeded XUV lasers. A home- 
made Velocity-Map-Imaging Spectrometer (VMIS), which provides photoelectron spectra with angular resolution, is the central element of the diagnostic ${ }^{12}$.

In this paper we report on the current progress in the development of our project. We have recently performed an experiment at the LASERIX facility (Universite Paris-Saclay, France), aimed to characterize the temporal profile of a seeded Ne-like Ti XUV laser emitting at $32.6 \mathrm{~nm}^{13}$, using the laser-dressed photoionization technique in the sideband regime with an IR dressing pulse. A measurement of the pulse duration of the seeded Ne-like Ti XUV laser was previously reported by Wang et al. ${ }^{14}$ several years ago, using an ultrafast X-ray streak camera. After deconvolution from the measured instrumental resolution of $1.5 \mathrm{ps}$, a pulse duration of $1.1+/-0.47 \mathrm{ps}$ was inferred. Compared to streak cameras, the laser-dressed photoionization technique has the potential to provide a better temporal resolution (of the order of the duration of the dressing pulse, $\sim 100 \mathrm{fs}$ ), a higher dynamic range (strongly limited by space-charge effects in the case of streak cameras) and to allow the determination of the absolute timing between the HH seed and the amplified output pulse. To the best of our knowledge no implementation of this technique with plasma-based XUV lasers has been reported in the literature until now. The specific features of these sources in comparison with XFELs or HH lead to additional challenges in the experimental implementation, as discussed at the end of in this paper.

In section 2 we recall the principle of the measurement and we present numerical simulations of the dressed photoelectron spectra, using a model based on the Strong Field Approximation (SFA) ${ }^{15}$. In section 3 we describe the experimental setup, which was implemented at LASERIX to ensure the temporal and spatial overlap of the XUV laser beam and the IR dressing beam in the interaction zone of the VMIS. In section 4 we present a preliminary analysis of our experimental data, showing the effect of the dressing field at a particular IR-XUV delay. Conclusions and directions for future work are summarized in section 5 .

\section{EXPERIMENTAL METHOD AND NUMERICAL SIMULATIONS}

For the purpose of our project we have built a custom velocity-map imaging spectrometer ${ }^{12}$, with a thick-lens configuration including 11 electrodes, following the design proposed by Kling et al. ${ }^{16}$. The principle of the VMI spectrometer consists in projecting with an intense electrostatic field the expanding sphere of photoelectrons, produced by the interaction of the XUV beam with a target gas, onto a position-sensitive detector: a microchannel plate coupled to a phosphor screen, then imaged on a CCD camera. Following image processing with an Abel-inversion procedure, the detected spectrum gives access to the distribution of kinetic energy of the photoelectrons with an angular resolution around the symmetry axis (polarization axis or beam propagation axis).

The left image in Fig. 1.a shows a calculated VMI spectrum, in which the photoline induced by XUV laser photons at 38 $\mathrm{eV}$ in argon ( $\mathrm{I}_{\mathrm{p}}=15.76 \mathrm{eV}$, including both $3 \mathrm{p}_{1 / 2}$ and $3 \mathrm{p}_{3 / 2}$ components) appears as a circle. The radius of the circle is given by the modulus of the photoelectron momentum $|\vec{p}|=\sqrt{p_{x}^{2}+p_{y}^{2}}=\sqrt{2 m_{e} . E_{c}}$, where $\mathrm{m}_{\mathrm{e}}$ is the electron mass and $E_{c}=h v-I_{p}$ is the kinetic energy of the photoelectron released from an atom with ionization potential $I_{p}$ by photons of energy hv. The spectrum can be transformed to a polar representation shown in the right image of Fig. 1.a, where the vertical axis is the angle $\theta$. Figure 1.b shows the angularly integrated spectrum.
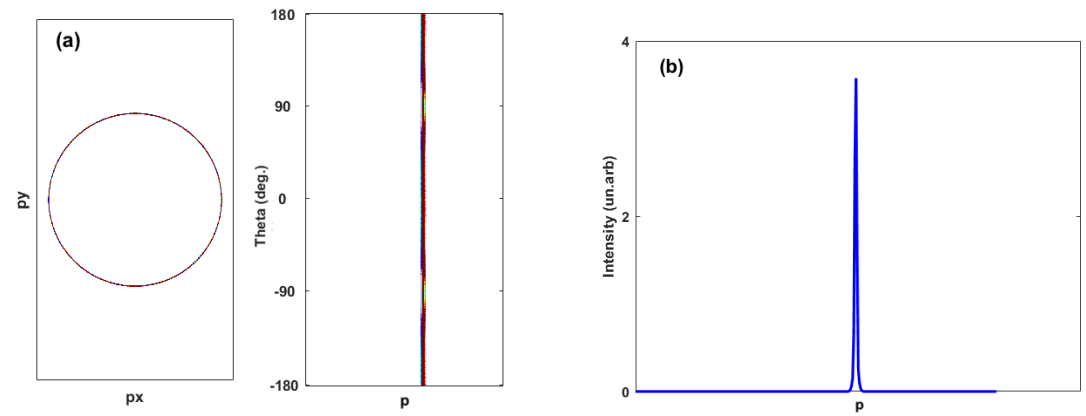

Figure 1. (a) Simulated photoelectron angular distribution, showing the single photoline corresponding to $5 \mathrm{p}$ ionization in argon with $38 \mathrm{eV}$ photons. Left: cartesian plot. Right: polar plot. (b) Angularly integrated spectrum showing the main photoline. 
Figure 2 shows how the angular distribution of photoelectrons is modulated when an IR dressing pulse is synchronized in time and overlapped in space with the XUV ionizing pulse. The numerical simulations were performed using a model based on the Strong Field Approximation ${ }^{15}$. The IR pulse, with a focused intensity of $2.10^{12} \mathrm{~W} / \mathrm{cm}^{2}$, is linearly polarized with its polarization axis parallel to the vertical $p_{y}$ direction. Up to four sideband peaks are visible on each side of the photoline, corresponding to the simultaneous absorption or stimulated emission of up to 4 IR photons. The sideband signal is maximum along the direction of polarization and is totally absent in the perpendicular direction. Figure $2 . b$ shows the angularly integrated spectrum, in which four sideband peaks are apparent. It is important to note, by comparing Fig. 1.b and Fig. 2.b (while noticing that their vertical scale are different), that the presence of the sideband peaks leads to a corresponding depletion of the main photoline intensity, since the number of photoelectrons remains constant.
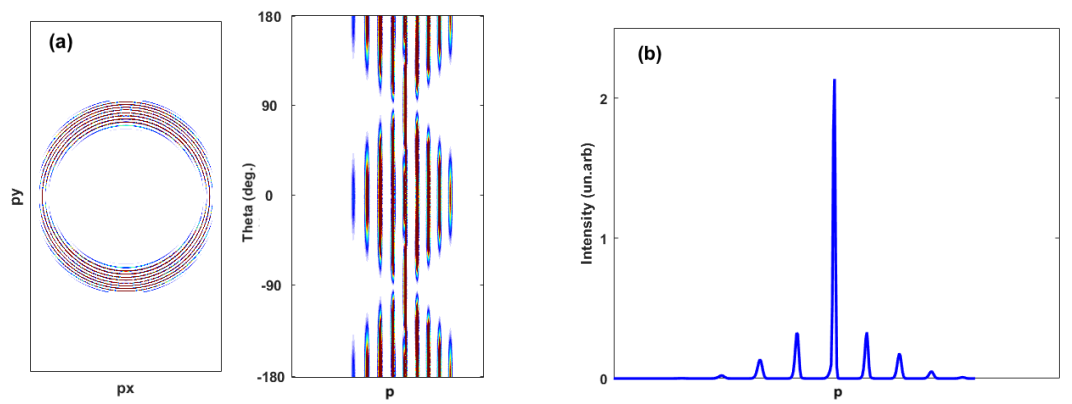

Figure 2. (a) Simulated photoelectron angular distribution, showing the sideband structures induced by an IR dressing pulse, synchronized with the XUV ionizing pulse, with intensity $2.10^{12} \mathrm{~W} / \mathrm{cm}^{2}$ and linear polarization in the vertical $\left(\mathrm{p}_{\mathrm{y}}\right)$ direction. Left: cartesian plot. Right: polar plot. (b) Angularly integrated spectrum showing the sideband peaks on each side of the main photoline.

Figure 2.a illustrates the potential benefit of using a VMI spectrometer for our temporal diagnostic, rather than a magnetic bottle or a time-of-flight spectrometer, with which the potentially rich information contained in the angular distribution of the photoelectrons is not accessible. A magnetic bottle spectrometer would lead to an angularly integrated spectrum, such as shown in Fig. 2.b. On the other hand a time-of-flight spectrometer would only detect a small portion (a few degrees) of the full angular distribution, at a given angle.

The number of sidebands and their relative intensity with respect to the main photoline are controlled by several parameters, among which the intensity of the IR dressing pulse, as well as its temporal delay and its duration relative to the XUV photoionizing pulse. Until now, the knowledge about the detailed temporal structure of seeded XUV lasers is provided by numerical simulations, based on a Maxwell-Bloch description of the amplified intensity ${ }^{2}$. Figure 3 shows an example of such a calculated profile. It is typically composed of three components with distinct timescales. The seeded amplified pulse follows the (unamplified) HH seed by a delay of the order of the dipole relaxation time $\tau_{\mathrm{d}}(\sim 1 \mathrm{ps})^{17}$. The pulse then exhibits few ultrashort ( $\sim 100 \mathrm{fs})$ oscillatory structures related to Rabi oscillations of the two atomic states interacting coherently with the resonant electromagnetic field. This oscillatory part is followed by a coherent wake with a timescale of the order of $\tau_{\mathrm{d}}$. The overall seeded amplified pulse is surrounded by a longer ASE background, with a duration, related to the gain lifetime, typically of the order of 5-10 ps. This ASE background pulse is difficult to avoid in seeded XUV lasers, while its level should be kept as small as possible in our measurement.



Figure 3. Calculated temporal structure of a seeded XUV laser, showing 3 components with different timescales (see text). 
For the numerical simulations of photoelectron spectra presented above the XUV ionizing pulse $(\mathrm{h} v=38 \mathrm{eV})$ was modeled by a short 30 fs Gaussian pulse representing the coherent seeded part, surrounded by a broader 300fs Gaussian pulse, representing the ASE background, with a 1:1 energy repartition in each pulse. The duration of the IR $(\lambda=800 \mathrm{~nm})$ dressing pulse was 15 fs. Durations shorter than the actual ones were used in order to reduce the computing time in our calculations. The simulation results presented here should thus be mainly considered as a support for a qualitative discussion.

Figure 4.a shows a spectrogram, i.e. the variation of the dressed photoelectron spectrum along the direction of polarization (i.e $\theta=0^{\circ}$ and $180^{\circ}$ in Fig 2.a), when changing the delay between the XUV pulse and the IR pulse (same parameters as above) from -150 fs to +150 fs. The $15 \mathrm{fs}$ IR pulse overlaps the $30 \mathrm{fs}$ XUV peak at zero delay. The number and intensity of the sidebands decrease on both sides of the zero delay, as the overlap between the XUV and dressing pulses decreases. Figure 4.b shows that by adding the intensity of all apparent sidebands for each delay, the resulting cross-correlation signal is close to the input XUV pulse profile.
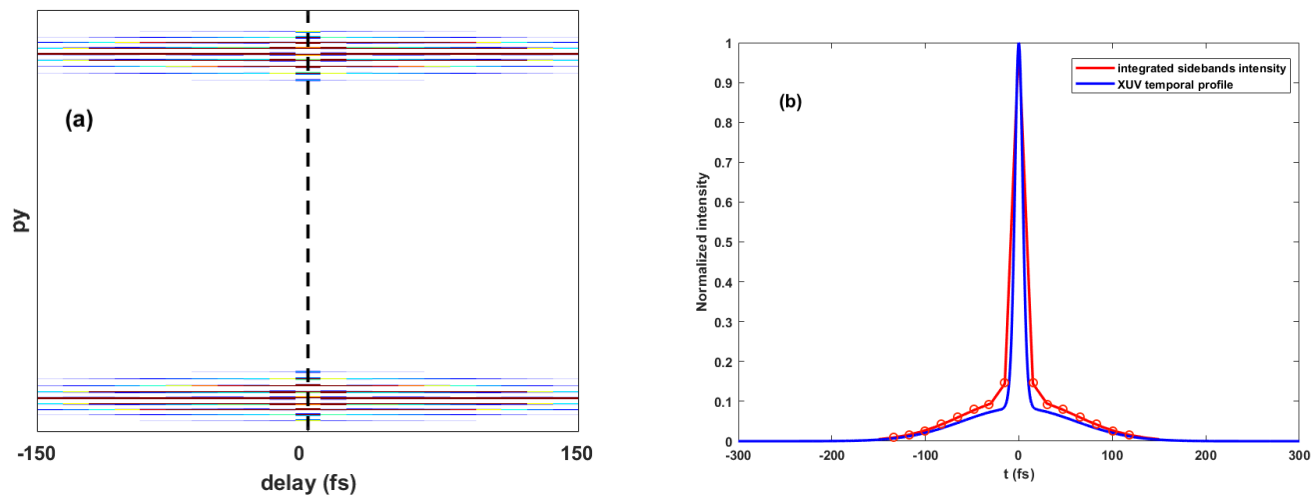

Figure 4. (a) Calculated spectrogram showing the evolution of the dressed photoelectron spectrum along the IR polarization direction, while varying the delay between the XUV and the IR dressing pulse by steps of $15 \mathrm{fs}$. (b) Integrated intensity of sidebands signal for the successive delay steps (red circles) compared to the input pulse profile (blue).

\section{EXPERIMENTAL SET-UP}

The experiment was carried out at the LASERIX facility, hosted at IJC-Lab, Université Paris-Saclay. Figure 5 shows the experimental setup. The seeded XUV laser beam (in pink) was generated in the grazing-incidence, transient pumping scheme by focusing on a titanium slab target (length $3 \mathrm{~mm}$ ) a sequence of 2 laser pulses: a $10 \mathrm{~ns}, 400 \mathrm{~mJ}$ pulse generated from a frequency-doubled Q-switched Nd YAG laser $(\lambda=532 \mathrm{~nm})$, followed after a delay of $4 \mathrm{~ns}$ by a $4 \mathrm{ps,} 1.2 \mathrm{~J}$ pulse generated from a Ti:Sa laser $(\lambda=815 \mathrm{~nm})$. The second pulse is focused on the target by a $500 \mathrm{~mm}$ spherical mirror used off-axis, leading to a grazing incidence pumping angle of $22^{\circ}$ at the target. For seeded operation using $\mathrm{H} 25$, the $\mathrm{HH}$ seed pulse is generated by focusing an auxiliary $40 \mathrm{fs}, 4 \mathrm{~mJ}$ infrared beam of in a $10 \mathrm{~mm}$ long Argon filled gas-cell. A more detailed description of the experimental conditions of the source is given in Ref. [17].

The XUV laser beam was transported and focused at the position of interaction in the VMIS using a $500 \mathrm{~mm}$ spherical multilayer (Mo:Si) mirror followed by a multilayer planar mirror, both used at near-normal incidence. The position of the spherical mirror was adjusted so as to produce a $\sim 10 \mathrm{x}$ magnified image of the XUV laser source to the center of the VMIS positioned at a distance of $5 \mathrm{~m}$. The IR dressing beam (in orange) was focused at the same position with a $\mathrm{f}=3 \mathrm{~m}$ spherical silica lens and reflected with a $45^{\circ}$ mirror to follow the path of the XUV beam with a small ( $\left.\sim 8 \mathrm{mrad}\right)$ angle. A variable aperture placed in front of the lens was used to adjust the energy/intensity of the dressing pulse.

The precise superposition in space and overlap in time of the XUV pulse and the IR dressing pulse are critical requirements of the experiment. The spatial superposition was controlled with a $\sim 30 \mu \mathrm{m}$ accuracy by sending both beams with a retractable mirror to an XUV CCD positioned at the same pathlength than the VMIS interaction zone (see Fig. 5). This camera was also used to determine the size of the focal spots in the VMIs interaction zone: typically $300 \mu \mathrm{mx} 500 \mu \mathrm{m}$ for the XUV beam, and $\sim 600 \mu \mathrm{m}$ diameter for the IR dressing beam. The temporal overlap was adjusted by observing interference fringes between the IR dressing pulse and the IR pulse used for the generation of the HH seed. These interference fringes were observed on a CCD camera placed along the propagation axis at the exit of the VMIS. From 
this position of temporal overlap, corresponding to delay $\Delta \mathrm{t}_{\mathrm{d}}=0$, the time of arrival of the dressing pulse in the VMIS interaction zone was varied with a delay line. During the experiment the duration of the dressing pulse was varied between $50 \mathrm{fs}$ (optimal compression) and 1 ps by changing the distance between the gratings of the compressor positioned before the delay line in the IR beam path.

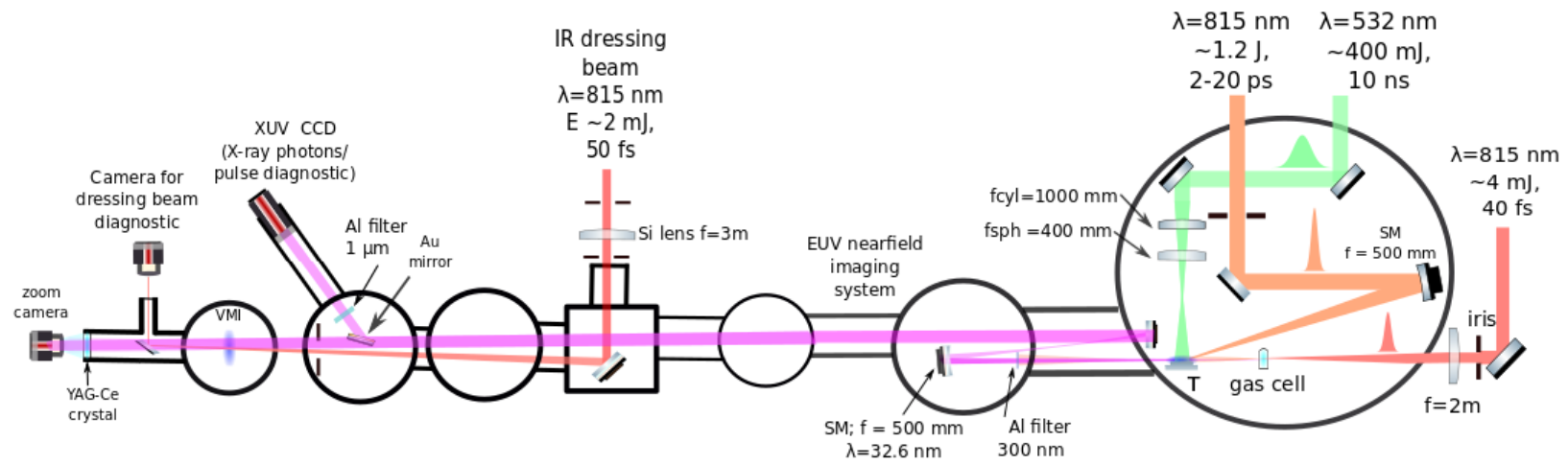

Figure 5. Experimental setup showing the seeded XUV laser generation chamber (right), as well as the XUV and IR beamlines used to transport and focus the beams to the VMI spectrometer (left). Additional diagnostics were implemented in order to control the spatial superposition and temporal overlap of the two pulses in the interaction region (see text).

As the generation of sidebands is also strongly dependent on the dressing intensity in the interaction region, several independent diagnostics were used to measure this parameter, as a function of the diameter of the beam (controlled with the variable aperture). In particular, we used the VMIS in the ion spatial mode, which allows to obtain a spatially resolved image of the ions produced in the interaction zone on the detector. By varying the diameter of the beam aperture we were able to assess the level of intensity above which ions were actually produced by the IR beam alone, as a result of multiphoton above-threshold-ionization (ATI) processes. Such ATI processes require a focused intensity of the order of ${ }^{18} 10^{13} \mathrm{~W} / \mathrm{cm}^{2}$. Figure 6 shows typical images obtained while increasing the diameter of the IR beam aperture. In each case the pulse energy was measured with a joulemeter and the size of the focal spot was inferred from the image taken with the XUV CCD. One can see that the trace of ATI processes appears at the position of the argon gas jet (which is also the position of beam focus) when the focused intensity reaches $1.910^{13} \mathrm{~W} / \mathrm{cm}^{2}$. For a larger intensity (bottom image) a weaker trace of ATI processes is also observed along the beam path, as the intensity is large enough to induce ATI in the residual gas close to the focus position. For the dressing measurements presented in section 4, the focused IR intensity was reduced to below $10^{13} \mathrm{~W} / \mathrm{cm}^{2}$.

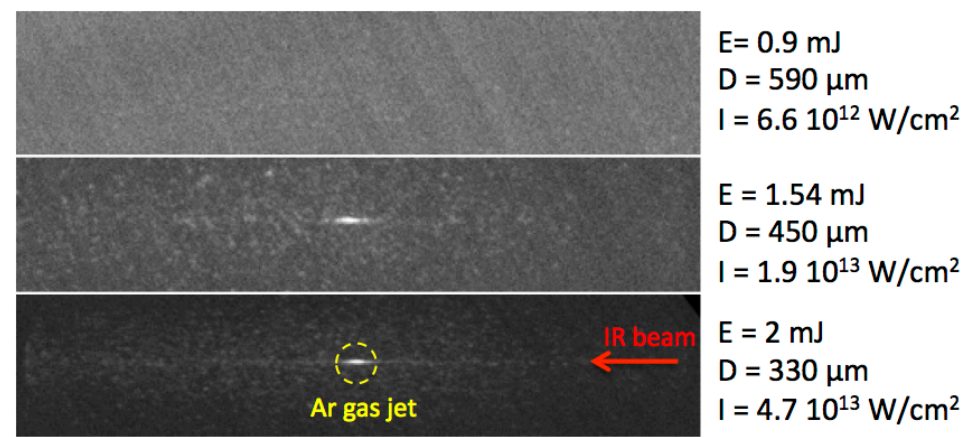

Figure 6. Spatial imaging of the argon ions produced in the VMIS by the dressing IR beam alone. The presence of ions at the position of the argon gas jet indicates that the focused intensity is large enough to induce multiphoton ATI processes.

\section{PRELIMINARY RESULTS}

During the experiment, carried out in November-December 2020, series of VMIS images were taken while varying several parameters of the IR dressing pulse: intensity, duration, and delay with respect to the time of HH injection. The data are still under analysis. In this section we show the first results of a preliminary analysis of the series of images taken for a duration of the dressing pulse enlarged to $900 \mathrm{fs}$. Such a long dressing pulse was used to increase the 
proportion of photoelectrons, which could "see" the dressing field, over those released before and after the dressing pulse over the duration of the ASE background. Other series were taken with shorter IR pulse durations, and at different dressing intensities.

Figure 7 (left) presents a raw photoelectron spectrum obtained from the VMIS, which shows a single photoline at $\mathrm{E}_{\mathrm{c}}=$ $22.24 \mathrm{eV}$, corresponding to the $3 p$ ionization of argon by the seeded XUV laser photons at $38 \mathrm{eV}$. The image is an average of ten individual images, each one being obtained from the accumulation of ten laser shots. For these shots, the dressing pulse was also present in the interaction zone with an intensity of $2.410^{12} \mathrm{~W} / \mathrm{cm}^{2}$. In the center of Fig. 7 , the same image is shown in a polar representation. As already explained in section 2, the effect of the laser dressing pulse is expected to be maximal along the polarization axis, i.e. at $\theta=0^{\circ}$ and $180^{\circ}$. The right image in Fig. 7 shows lineouts of the signal taken along the horizontal direction at $\theta=180^{\circ}$ for two different values of the XUV-IR delay $\left(\Delta \mathrm{t}_{\mathrm{d}}=600 \mathrm{fs}\right.$ and $900 \mathrm{fs}$ ). We remind that $\Delta \mathrm{t}_{\mathrm{d}}=0$ corresponds to the IR dressing pulse synchronized with the IR pulse that generates the $\mathrm{HH}$ seed, at the position of the interaction zone in the VMIS. A lineout corresponding to an averaged image obtained from shots with the XUV pulse only (no IR dressing pulse) is shown for comparison. For each lineout the signal was first normalized to the radius-averaged signal at $\theta=+/-90^{\circ}$ (i.e. in the direction perpendicular to the polarization axis). The black arrow indicates the position of the first sideband at $\mathrm{E}_{\mathrm{c}}=22.24+1.55=23.79 \mathrm{eV}$.



Figure 7. Left: Raw photoelectron spectrum (average of 10 images, each one obtained by accumulating 10 shots) corresponding to the $5 \mathrm{p}$ ionization of argon by $38 \mathrm{eV}$ photons. The red arrow indicates the direction of polarization of the IR dressing pulse (duration $900 \mathrm{fs}$, intensity $2.610^{12} \mathrm{~W} / \mathrm{cm}^{2}$ ). Center: Same spectrum in polar representation. Right: Lineouts of the signal at $\theta=180^{\circ}$ (as shown by the yellow dotted line in the center image) for two different XUV-IR delays $\Delta \mathrm{t}_{\mathrm{d}}$ and for the case without IR dressing pulse. The black vertical arrow indicates the position of the first sideband peak at $\mathrm{E}_{\mathrm{c}}=23.79 \mathrm{eV}$.

One can see that for the two delays shown, the signal with the IR dressing pulse is slightly higher at this position and correlatively weaker at the position of the main photoline. The resolution of the VMIS is not sufficient to fully resolve the sideband peak, so the sideband peaks is blended with the main photoline. A similar wing feature is also observed for lineouts taken at $\theta=180^{\circ}$. We believe that this feature is related to the effect of IR dressing. The fact that this effect is observed for $\Delta \mathrm{t}_{\mathrm{d}}=600 \mathrm{fs}$ and $900 \mathrm{fs}$ and not at shorter or longer delays would be consistent with the delay between the HH seed and the amplified coherent pulse predicted from the simulations (see Figure 3). Again we emphasize that these results are still preliminary and have to be confirmed by a more complete treatment/analysis of the series of experimental data. The angular resolution in the experimental data provide more robustness in the reliability of their interpretation, as any effect of the IR laser dressing pulse should be observed in a similar way along the IR polarization axis $\left(\theta=0^{\circ}\right.$, $\left.180^{\circ}\right)$, while being absent in the perpendicular direction $\left(\theta=+/-90^{\circ}\right)$.

\section{CONCLUSION}

In conclusion, we have reported on the first implementation of a velocity-map imaging spectrometer to characterize the temporal structure of a seeded XUV laser using the technique of laser-dressed photoionization in the sideband regime. This technique was already demonstrated with other ultrashort XUV sources, namely gas high-order harmonics and XUV free-electron lasers. The principle of the method was investigated using numerical simulations in the strong field approximation. The experimental implementation of the diagnostic with seeded XUV lasers is found to be challenging, because the observation of the sideband peaks induced by the IR dressing pulse is partially hindered by several source features, in particular the presence of a long ASE background pulse, or the contribution of incoherent thermal plasma 
emission. A preliminary analysis of the experimental data recently obtained at the LASERIX facility suggests that the effect of the dressing pulse is observed in the photoelectron spectra at specific delays between the XUV pulse and the IR dressing pulse. A more detailed and complete treatment of the series of data is in progress.

\section{ACKNOWLEDGEMENTS}

This project has received funding from Labex PALM (ANR-10-LABX-0039-PALM), LIDEX OPT2X, Sesame PULSEX, Laserlab-Europe (EU-H2020 871124). The technical support of engineers and technicians, in particular J. Guigand, C. Charrière, N. Tournier at ISMO, and J. Demailly at IJC-Lab, is gratefully acknowledged.

\section{REFERENCES}

[1] Wang, Y., et al., "High-Brightness Injection-Seeded Soft-X-Ray-Laser Amplifier Using a Solid Target. ”, Phys. Rev. Lett., 97(12),1-4 (2006).

[2] Larroche, O., \& Klisnick, A., "Two-dimensional Maxwell-Bloch simulation of quasi- $\pi$-pulse amplification in a seeded XUV laser.”, Phys. Rev. A, 88 (3), 033815 (2013).

[3] Düsterer, S., et al. "Development of experimental techniques for the characterization of ultrashort photon pulses of extreme ultraviolet free-electron lasers.", Phys. Rev. S. T., 17(12), 120702 (2014).

[4] Orfanos, I., Makos, I., Liontos, I., Skantzakis, E., Förg, B., Charalambidis, D., \& Tzallas, P., "Attosecond pulse metrology.", APL Photonics, 4, 080901 (2019)

[5] Radcliffe, P., et al., "Atomic photoionization in combined intense XUV free-electron and infrared laser fields.", New J. Phys., 14, 043008 (2012).

[6] Azima, A., et al., "Direct measurement of the pulse duration and frequency chirp of seeded XUV free electron laser pulses.", New J. Phys., 20(20) (2018)

[7] Finetti, P., et al. "Pulse Duration of Seeded Free-Electron Lasers", Phys. Rev. X, 7, 21043 (2017)

[8] Düsterer, S., et al. "Femtosecond X-ray pulse length characterization at the Linac Coherent Light Source freeelectron laser.", New J. Phys., 13, 93024 (2011)

[9] Bouhal, A. et al., "Cross-Correlation Measurement of Femtosecond Noncollinear High-Order Harmonics", J. Opt. Soc.B, 14, 4, 950 (1997)

[10] Paul P., et al., "Observation of a train of attosecond pulses from high harmonic generation", Science 292, 16891692 (2001).

[11] Goulielmakis, E., et al., "Single-cycle nonlinear optics", Science, 320, 1614-1617 (2008)

[12] Bourassin-Bouchet C., et al., "Single-shot temporal characterization of ultrashort XUV pulses", X-ray Lasers and Coherent X-ray sources: Development and Applications XIII Proc. of SPIE, Vol. 11111, 111110E (2019)

[13] Guilbaud, O., et al., "Gain dynamics in quickly ionized plasma for seeded operated soft x-ray lasers", Opt. Lett., 40, 4775-4778 (2015)

[14] Wang, Y., et al., "Measurement of 1-ps soft-x-ray laser pulses from an injection-seeded plasma amplifier", Phys. Rev. A, 79(2), 1-4 (2009).

[15] Kazansky A K, Sazhina I P and Kabachnik N M, "Angle-resolved electron spectra in short-pulse two-color XUV+IR photoionization of atoms", Phys. Rev. A, 82, 033420 (2010)

[16] Kling N.G., et al., "Thick-lens velocity-map imaging spectrometer with high resolution for high-energy charged particles", J. Inst., 9, P05005 (2014)

[17] Kumar-Pandey A., et al., "Towards subpicosecond pulses from solid target plasma based seeded soft X-ray laser”, Opt. Expr., 28(20), 28924-28941 (2020).

[18] Cormier, E., et al. "Above-threshold ionization contrast and channel closure in argon.”, J. Phys. B, 34, L9-L17 (2001) 